\title{
Different distributions of M1 and M2 macrophages in a mouse model of laser-induced choroidal neovascularization
}

\author{
YEDI ZHOU, SHIGEO YOSHIDA, YUKI KUBO, TAKERU YOSHIMURA, YOSHIYUKI KOBAYASHI, \\ TAKAHITO NAKAMA, MUNEO YAMAGUCHI, KEIJIRO ISHIKAWA, YUJI OSHIMA and TATSURO ISHIBASHI
}

Department of Ophthalmology, Graduate School of Medical Sciences, Kyushu University, Fukuoka 812-8582, Japan

Received November 8, 2015; Accepted November 4, 2016

DOI: $10.3892 / \mathrm{mmr} .2017 .6491$

\begin{abstract}
Choroidal neovascularization (CNV) is a serious complication of age-related macular degeneration. The aim of the present study was to investigate the expression and distribution of M1 and M2 macrophages in a laser-induced CNV adult mouse model. The mRNA expression levels of M1, M2 and pan macrophage markers, and macrophage-associated angiogenic cytokines, were determined by reverse transcription-quantitative polymerase chain reaction. Immunofluorescence studies were performed to determine the location of the macrophages. The expression levels of M1 macrophage markers increased to a greater extent compared with M2 markers in the retinal pigment epithelium (RPE)-choroid complexes following laser photocoagulation. By contrast, the expression levels of M2 macrophage markers increased primarily in the retinas. Immunofluorescence studies revealed that the increased number of cluster of differentiation (CD)206-positive cells were located primarily in the retina, whereas the CD80-positive cells were located around the site of CNVs in the RPE-choroid. In addition, the M1-associated cytokines increased to a greater extent in the RPE-choroid complexes, whereas the M2-associated cytokines were highly expressed in the retinas. These findings indicate that M1 and M2 macrophage numbers increased following CNV; however, the locations were different in this mouse model of laser-induced CNV. The results of the present study suggest that M1 macrophages have a more direct role in inhibiting the development of CNV.
\end{abstract}

\section{Introduction}

Age-related macular degeneration (AMD) is a leading cause of blindness in developed countries $(1,2)$. Choroidal

Correspondence to: Dr Shigeo Yoshida, Department of Ophthalmology, Graduate School of Medical Sciences, Kyushu University, 3-1-1 Maidashi, Higashi-ku, Fukuoka 812-8582, Japan

E-mail: yosida@med.kyushu-u.ac.jp

Key words: M1 macrophage, M2 macrophage, choroidal neovascularization, angiogenesis neovascularization (CNV) is a damaging complication of AMD (3); however, the exact mechanism underlying the development of $\mathrm{CNV}$ has not been determined. The laser-induced CNV mouse model is a well-known model of $\mathrm{CNV}$, which has been widely used to determine the underlying mechanisms involved in its development (4). In this model, the depletion of macrophages by clodronate liposomes leads to a reduction in the size of the induced CNVs, indicating that macrophages are essential for their formation and progression $(5,6)$. In addition, it has been reported that blood-derived F4/80-positive macrophages infiltrate the retina and activate Müller cells under the CNVs in this model (7). This indicates that the recruited blood-derived macrophages associate with the laser-induced CNV rather than the resident microglia.

There are two subtypes of macrophages, M1 and M2 $(8,9)$. M1, or pro-inflammatory macrophages, are considered to be important for the destruction of tumor cells and foreign organisms, whereas M2, or anti-inflammatory macrophages, have been suggested to be primarily involved in angiogenesis, wound healing, chronic infections, tumorigenesis and tumor metastasis (10-14). It has been suggested that the pathological shift of macrophage polarization may contribute to the pathogenesis of AMD (15).

It has been reported that M1 and M2 macrophages express different levels of angiogenic cytokines and growth factors (14). M2 macrophages have been revealed to promote angiogenesis in in vivo studies $(14,16)$. In addition, it has been demonstrated that more M2-like macrophages accumulate at the site of wet compared with dry AMD (15), suggesting that M2 macrophages may be involved in the development of CNVs. M2 macrophages injected into the eyes of mice promoted the progression of CNV lesions, whereas M1 macrophages inhibited them. These findings indicated that these macrophage subtypes have a causal role in AMD (17). The number of cluster of differentiation (CD)80-positive M1 macrophages and CD206-positive M2 macrophages have been demonstrated to be increased in the posterior segment of eyes in a CNV model (17). However, the exact locations of M1 and M2 macrophages in eyes with CNVs remain undetermined.

The aim of the present study was therefore to investigate the expression and distribution of the M1 and M2 macrophages in a mouse model of laser-induced CNV. 


\section{Materials and methods}

Animals. A total of 50 male C57BL/6 J mice (age, 6-9 weeks) were purchased from Kyudo (Tosu, Japan) were used in the present study. All mice were housed in a pathogen-free facility with access to food and water under controlled conditions (temperature, $23 \pm 1^{\circ} \mathrm{C}$; humidity, $55 \%$; 12 -h light/dark cycle). All experimental procedures were approved by the Committee on the Ethics of Animal Experiments of Kyushu University Graduate School of Medical Sciences (Fukuoka, Japan), and animals were cared for according to the Association for Research in Vision and Ophthalmology Statement for the Use of Animals in Ophthalmic and Vision Research.

Laser-induced CNV mouse model. Prior to the laser-treatment, mice were anesthetized by an intraperitoneal injection of a mixture of ketamine (Ketalar; $80 \mathrm{mg} / \mathrm{kg}$; Daiichi-Sankyo, Co., Ltd., Tokyo, Japan) and xylazine (Selactar; $10 \mathrm{mg} / \mathrm{kg}$; Bayer AG, Leverkusen, Germany). CNV was induced by laser photocoagulation as previously described $(18,19)$. In brief, the photocoagulations were placed around the optic disc with a 532-nm diode laser (200 mW, $0.1 \mathrm{sec}$ duration, $75 \mu \mathrm{m}$; Verdi; Coherent, Inc., Santa Clara, CA, USA). A total of five spots were burned in each eye; four for observation and one for orientation.

Prior to sacrifice, mice were anesthetized as above. The mice were sacrificed by cervical dislocation at 1-7 days after laser-treatment. The retinas were isolated from the posterior segment of the eyes, and flat-mounted for immunofluorescence studies. For reverse transcription-quantitative polymerase chain reaction (RT-qPCR), 20 spots were burned on each eye, and the eyes were enucleated at various time-points following laser photocoagulation.

$R T-q P C R$ analysis. RT-qPCR was performed as described previously $(16,20)$. Total RNA was extracted from the retinal pigment epithelium (RPE)-choroid complexes or homogenized retinas at the selected time-points. The MagDEA ${ }^{\circledR}$ RNA kit (Precision System Science USA, Inc., Pleasanton, CA, USA) was used to extract RNA according to the manufacturer's protocol. RNA concentrations were measured and cDNA was subsequently synthesized using a First Strand cDNA Synthesis kit (Roche Diagnostics GmbH, Mannheim, Germany). qPCR was performed using the TaqMan ${ }^{\circledR}$ gene expression assays listed below (Applied Biosystems; Thermo Fisher Scientific, Inc., Waltham, MA, USA) and a LightCycler ${ }^{\circledR} 96$ Real-Time PCR system (Roche Diagnostics GmbH).

CD80 and CD86 were used as M1 macrophage markers (21), and CD206 and CD163 were used as M2 macrophage markers $(22,23)$, and F4/80 was used as a pan macrophage marker. The reference numbers for the assays were as follows: Mm99999915_g1 (GAPDH), Mm00802529_m1 (F4/80), Mm00485148_m1 (CD206), Mm00474091_m1 (CD163), Mm00711660_m1 (CD80), Mm00444543_m1 (CD86), Mm00433287_m1 [basic fibroblast growth factor (Fgf2)], Mm00435613_m1 [placental growth factor (Pgf)], Mm00449032_g1 [thrombospondin 1 (Thbs1)] and Mm00441242_m1 [monocyte chemoattractant protein-1 (Ccl2)]. GAPDH served as an endogenous control. For the TaqMan assays, the HotStart DNA polymerase was activated by an initial 10 -min incubation at $95^{\circ} \mathrm{C}$, followed by 45 cycles of $95^{\circ} \mathrm{C}$ for $20 \mathrm{sec}$ and $60^{\circ} \mathrm{C}$ for $40 \mathrm{sec}$. The detection of the probe, calculation of quantitation cycles (24) and further analysis were performed using the LightCycler ${ }^{\circledR}$ 96 Real-Time PCR system software (Roche Diagnostics $\mathrm{GmbH})$. Four samples were examined in each group.

Immunofluorescence. Immunofluorescence staining was performed as previously described $(16,18,25)$, with certain minor modifications. Briefly, eyes were enucleated and fixed in $4 \%$ paraformaldehyde for $1 \mathrm{~h}$, the corneas and muscles were removed, and the posterior segments were placed in $4 \%$ paraformaldehyde for a further $1 \mathrm{~h}$. The lens, uvea and sclera were excised, and the retina was isolated from certain posterior segments. For other eyes, $20 \mu \mathrm{m}$ sections were cut with a cryostat (Leica CM1800; Leica Microsystems, Inc., Buffalo Grove, IL, USA). Following rinsing and blocking, the posterior segment of the eyes, isolated retinas or cryostat sections were incubated with the primary or conjugated antibodies overnight at $4^{\circ} \mathrm{C}$. The following secondary antibodies $(1: 200)$ were added for $1 \mathrm{~h}$ at room temperature to detect anti-CD31 binding: Alexa Fluor $^{\circledR} 488$ chicken anti-goat IgG (catalog no. A-21467), Alexa Fluor 546 donkey anti-goat IgG (catalog no. A-11056) or Alexa Fluor 647 chicken anti-goat IgG (catalog no. A-21469), all obtained from Thermo Fisher Scientific, Inc. Hoechst 33342 (Molecular Probes; Thermo Fisher Scientific, Inc.) was used to counterstain the nuclei in the cryostat sections. Following rinsing with phosphate buffered saline containing Tween-20 20 , the cryostat sections and flat-mounts were coverslipped using a PermaFluor aqueous mounting medium (Thermo Fisher Scientific, Inc.).

The samples were incubated with the following primary antibodies: Alexa Fluor 647 anti-mouse CD206 (1:100; catalog no. 141712; BioLegend, Inc., San Diego, CA, USA), fluorescein isothiocyanate anti-mouse CD80 (1:200; catalog no. 11-0801; eBioscience, Inc., San Diego, CA, USA), fluorescein-labeled isolectin B4 (1:150; catalog no. FL-1201; Vector Laboratories, Inc., Burlingame, CA, USA), DyLight ${ }^{\circledR}$ 594-labeled isolectin B4 (1:150; catalog no. DL-1207; Vector Laboratories, Inc.) or goat anti-mouse CD31 (1:20; catalog no. AF3628; R\&D Systems, Inc., Minneapolis, MN, USA). The sections and flat-mounts were analyzed under a BZ-9000 fluorescence microscope (Keyence Corporation, Osaka, Japan) $(20,26,27)$. Posterior segments of the eyes, containing connected retina and choroid were examined and analyzed using a laser scanning confocal microscope (Nikon Corporation, Tokyo, Japan) to obtain three-dimensional images.

Statistical analysis. All results are presented as the mean \pm standard error. Differences between groups were compared using Dunnett's tests or Student's t-tests. $\mathrm{P}<0.05$ was considered to indicate a statistically significant difference. Statistical analyses were performed using JMP software version 10.0.2 (SAS Institute, Cary, NC, USA).

\section{Results}

Recruitment of M1 macrophages to RPE-choroid complexes of $C N V$. RT-qPCR was performed to determine whether M1 and M2 macrophages were associated with the development of 
A

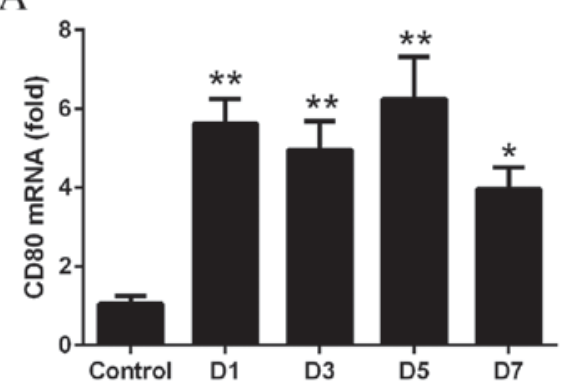

C

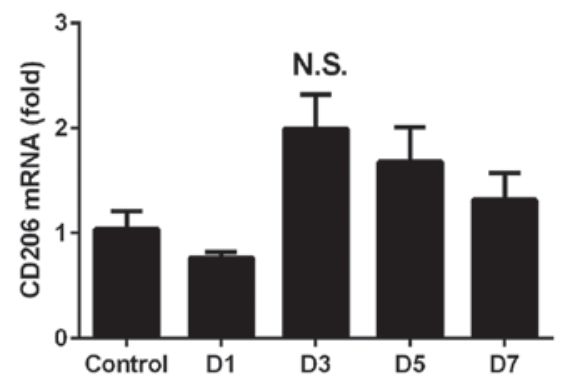

E

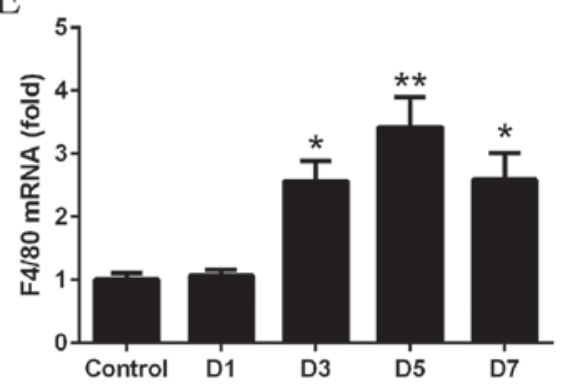

B

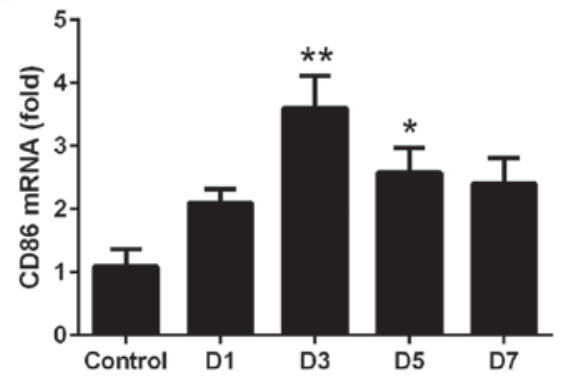

D

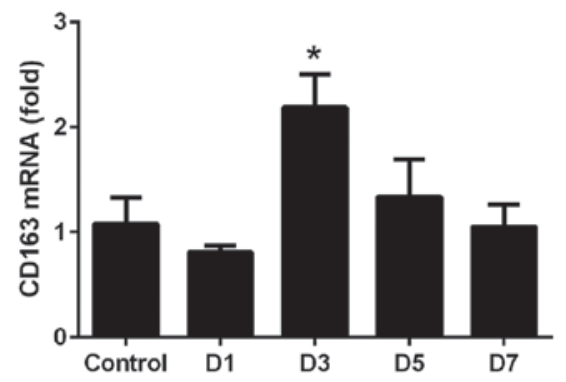

Figure 1. mRNA expression levels of macrophage markers, as determined by reverse transcription-quantitative polymerase chain reaction. Retinal pigment epithelium-choroid complexes were harvested from mice 1 to 7 days following laser-induced choroidal neovascularization. The mRNA expression levels of (A) CD80 and (B) CD86 were significantly increased following laser treatment, compared with control untreated tissues. No significant differences were observed in the mRNA expression levels of (C) CD206, and the levels of (D) CD163 were significantly increased only at day 3. The mRNA expression levels of (E) F4/80 were significantly increased at days 3 to 7 . Data are expressed as the mean \pm standard error $(n=4)$. ${ }^{* *} \mathrm{P}<0.01$ and ${ }^{*} \mathrm{P}<0.05$ vs. control. $\mathrm{CD}$, cluster of differentiation; D, day; NS, non-significant.

laser-induced CNV. The mRNA expressions levels of CD80, CD86, CD206, CD163 and F4/80 in the RPE-choroid complex at days 1-7 following laser treatment were compared with those in the untreated control tissues. The mRNA expression levels of $\mathrm{CD} 80$ (day 1, $\mathrm{P}=0.0011$; day $3, \mathrm{P}=0.0041$; day 5, $\mathrm{P}=0.0003$; day 7, $\mathrm{P}=0.0311$; Fig. $1 \mathrm{~A}$ ) and $\mathrm{CD} 86$ (day 3, $\mathrm{P}=0.0009$; day 5, $\mathrm{P}=0.0418$; Fig. 1B) were significantly upregulated following laser treatment. The mRNA expression levels of the M2 markers CD206 (P>0.05; Fig. 1C) and CD163 (day 3, P=0.0291; Fig. 1D) were increased to a lesser extent. The mRNA expression levels of F4/80 increased from days 3 to 7, with the degree of increase between that of the M1 and M2 markers (day 3, $\mathrm{P}=0.013$; day 5, $\mathrm{P}=0.0003$; day 7, $\mathrm{P}=0.0119$; Fig. 1E).

Recruitment of $M 2$ macrophages in retinas with $C N V$. To determine whether M1 and M2 macrophages were recruited into the retinas, RT-qPCR was performed on the retinas at days 1-7 following laser treatment. No significant differences were observed in the mRNA expression levels of CD80 at anytime point $(\mathrm{P}>0.05$; Fig. 2A). However, the expression levels of CD86 were significantly upregulated on day 5
( $\mathrm{P}=0.0024$; Fig. 2B). The mRNA expression levels of CD206 (day 3, $\mathrm{P}=0.0427$; day 5, $\mathrm{P}=0.0077$; Fig. 2C) and CD163 (day 5, $\mathrm{P}=0.0497$; Fig. 2D) were significantly increased, with the greatest fold-changes observed on days 3 and 5. The mRNA expression levels of F4/80 significantly increased from days 3 to 5, with the degree of increase between that of M1 and M2 (day 3, P=0.0138; day 5, P=0.0004; Fig. 2E).

\section{Predominance of M1 macrophages in choroid and M2} macrophages in retina following laser-induced $C N V$. To determine the distributions of M1 and M2 macrophages in the retina, double immunofluorescence staining of cryosections was performed on day 3 following laser photocoagulation using antibodies against CD31 and CD80 or CD206. The CD80-positive cells were located around the site of laser-photocoagulations (Fig. 3A). By contrast, numerous CD206-positive cells were detected in the inner layer of the retina around the CD31-positive vessels (Fig. 3B).

Double immunofluorescence staining with isolectin B4 and CD80 or CD206 was additionally performed in flat-mounted retinas and choroid following laser photocoagulation. Numerous 
A

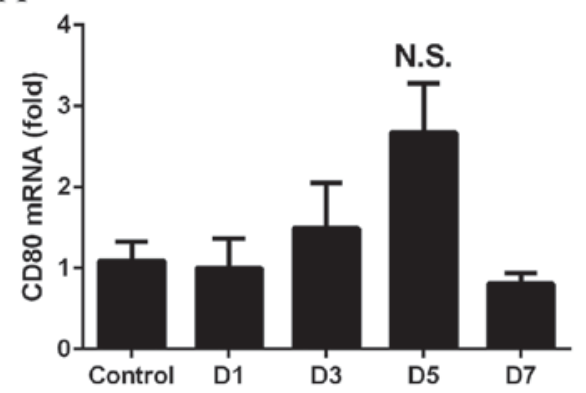

C

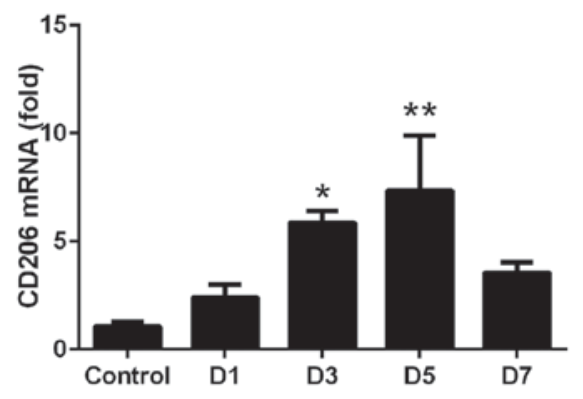

E

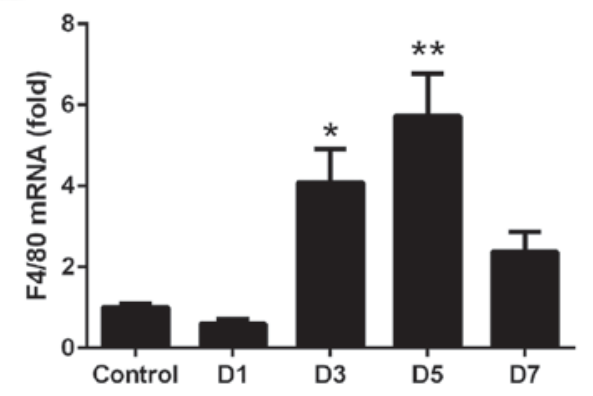

B

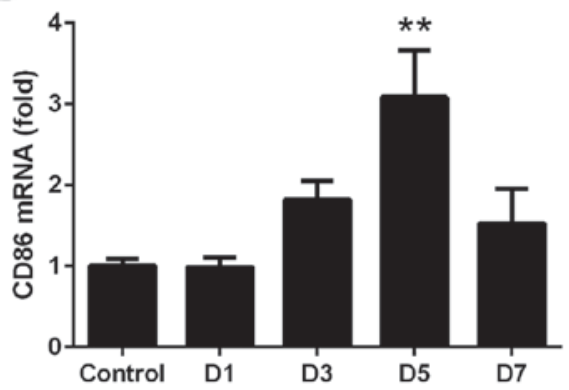

D

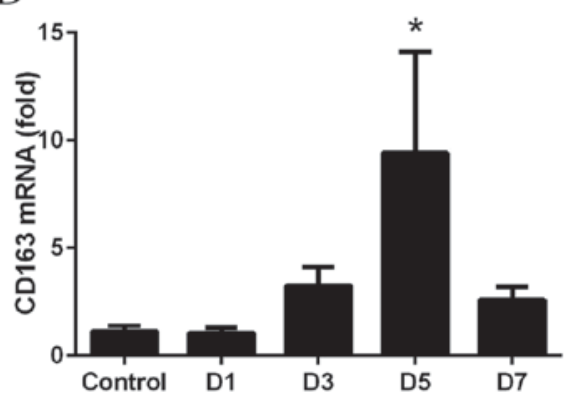

Figure 2. mRNA expression levels of macrophage markers, as determined by reverse transcription-quantitative polymerase chain reaction. Retinas were harvested from mice 1 to 7 days following laser-induced choroidal neovascularization. No significant differences were observed in the mRNA expression levels of (A) CD80 following laser treatment, compared with control untreated tissues; however, the mRNA expression levels of (B) CD86, (C) CD206, (D) CD163 and (E) F4/80 significantly increased in response to laser treatment. Data are expressed as the mean \pm standard error $(\mathrm{n}=4)$. ${ }^{* *} \mathrm{P}<0.01$ and ${ }^{*} \mathrm{P}<0.05$ vs. control. $\mathrm{CD}$, cluster of differentiation; D, day; NS, non-significant.

CD80-positive cells were observed in the laser-injured areas (Fig. 3C); however, very few CD206-positive cells were present (Fig. 3D). By contrast, the number of CD206-positive cells increased markedly in laser-treated retinas compared with controls (Fig. 3E and F).

Distribution of M1 and M2 macrophages in three dimensional images. To further confirm the differential distribution of M1 and M2 macrophages in the retina and choroid, triple immunofluorescence staining was performed in the posterior segments of the eyes, with intact retina and choroid. Consistent with the findings presented in Fig. 3, CD80-positive M1 macrophages were observed in and around the CNV lasered areas. By contrast, the majority of the CD206-positive M2 macrophages were observed in the upper layer of the retina (Fig. 4).

mRNA expression levels of M1 and M2 macrophage-associated cytokines in RPE-choroid complex and retina following $C N V$. To determine the synthesis of cytokines by M1 and M2 macrophages in the laser-induced CNV model, RT-qPCR was performed to examine the mRNA expression levels of various angiogenic factors using cDNA from the retina and RPE-choroid complex obtained at day 3 following laser treatment. The mRNA expression levels of Thbs1 increased in the RPE-choroid complex following CNV ( $\mathrm{P}=0.0026$; Fig. 5A) compared with control untreated tissues, whereas Pgf decreased significantly ( $\mathrm{P}=0.0006$; Fig. 5B). mRNA expression levels of Fgf2 ( $\mathrm{P}=0.0132$; Fig. 5C) and $\mathrm{Ccl} 2$ ( $\mathrm{P}=0.0002$; Fig. 5D) significantly increased in the RPE-choroid complex following laser treatment. In the retina, no significant differences were observed in Thbs1 mRNA expression levels (Fig. 5E); however, the mRNA expression levels of $\mathrm{Pgf}(\mathrm{P}=0.0006$; Fig. $5 \mathrm{~F})$, Fgf2 ( $\mathrm{P}=0.0009$; Fig. 5G) and $\mathrm{Ccl} 2(\mathrm{P}=0.0060$; Fig. 5H) all increased significantly in the laser-treated compared with control untreated tissues.

\section{Discussion}

It has recently been reported that the number of macrophages increases in choroid following laser photocoagulation in mice (4). The results of the present study demonstrated that the mRNA expression levels of M1 and M2 macrophage 

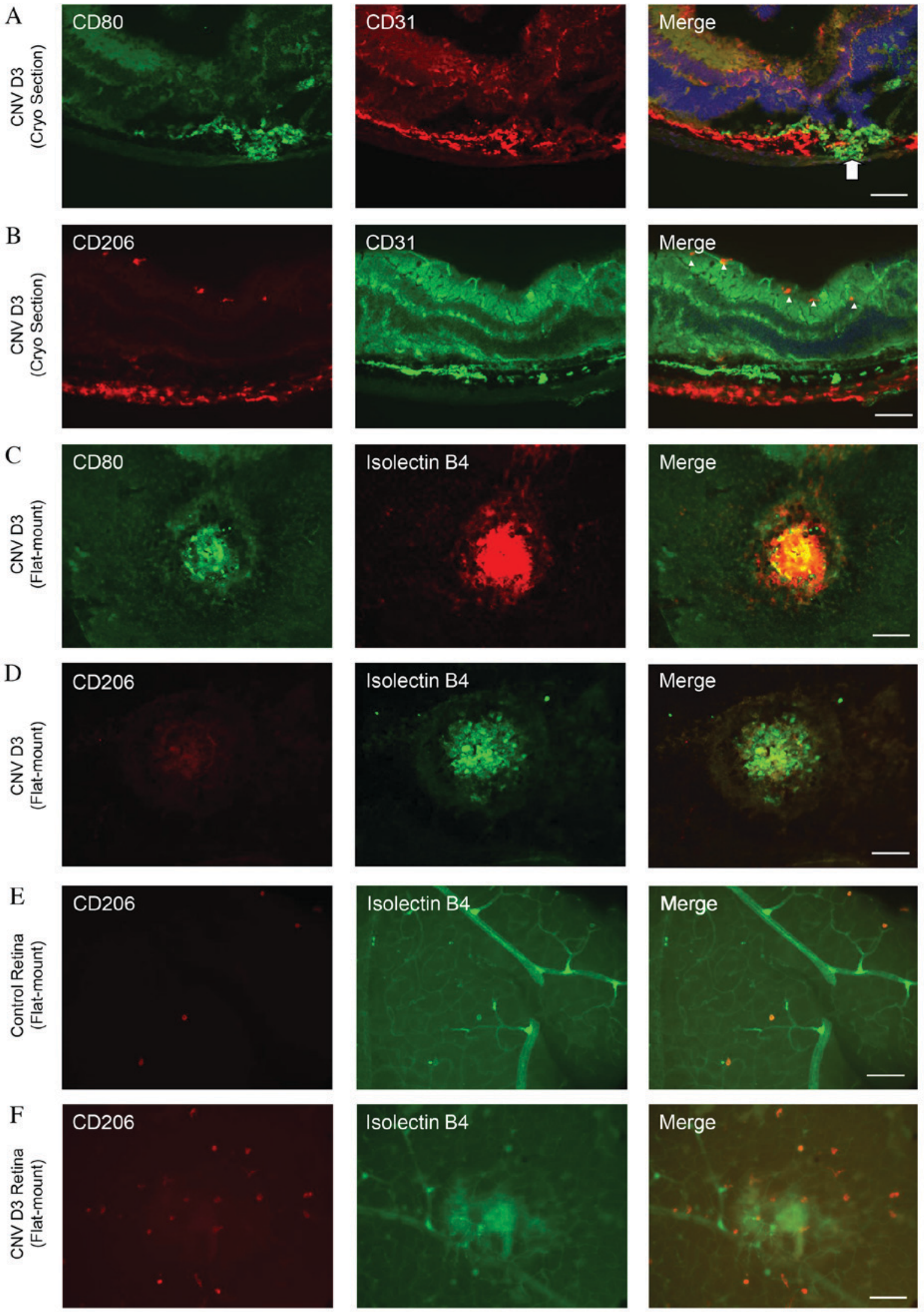

Figure 3. Distribution of M1 and M2 macrophages in a mouse model of laser-induced CNV. (A) Double staining for CD80 (green) and CD31 (red) in cryosections of CNV eyes at day 3. CD80-positive cells were located close to the site of laser injury, as indicated by the arrow. (B) Double staining for CD206 (red) and CD31 (green) in cryosections of CNV eyes at day 3. The majority of the CD206-positive cells were colocalized with CD31-positive cells in the inner layer of the retina, as indicated by the arrowheads. (C) Double staining for CD80 (green) and isolectin B4 (red) in flat-mounted choroid of CNV eyes at day 3. Certain CD80-positive cells were colocalized with isolectin B4-positive areas around the site of the laser injury. (D) Double staining for CD206 (red) and isolectin B4 (green) in flat-mounted choroid of CNV eyes at day 3. Very few CD206-positive cells were detected. Double staining for CD206 (red) and isolectin B4 (green) in flat-mounted retina of (E) control and (F) CNV eyes at day 3. A greater number of CD206-positive cells were observed in the retinas of CNV, compared with control, eyes. Scale bars, $100 \mu \mathrm{m}$. CNV, choroidal neovascularization; CD, cluster of differentiation; D, day. 

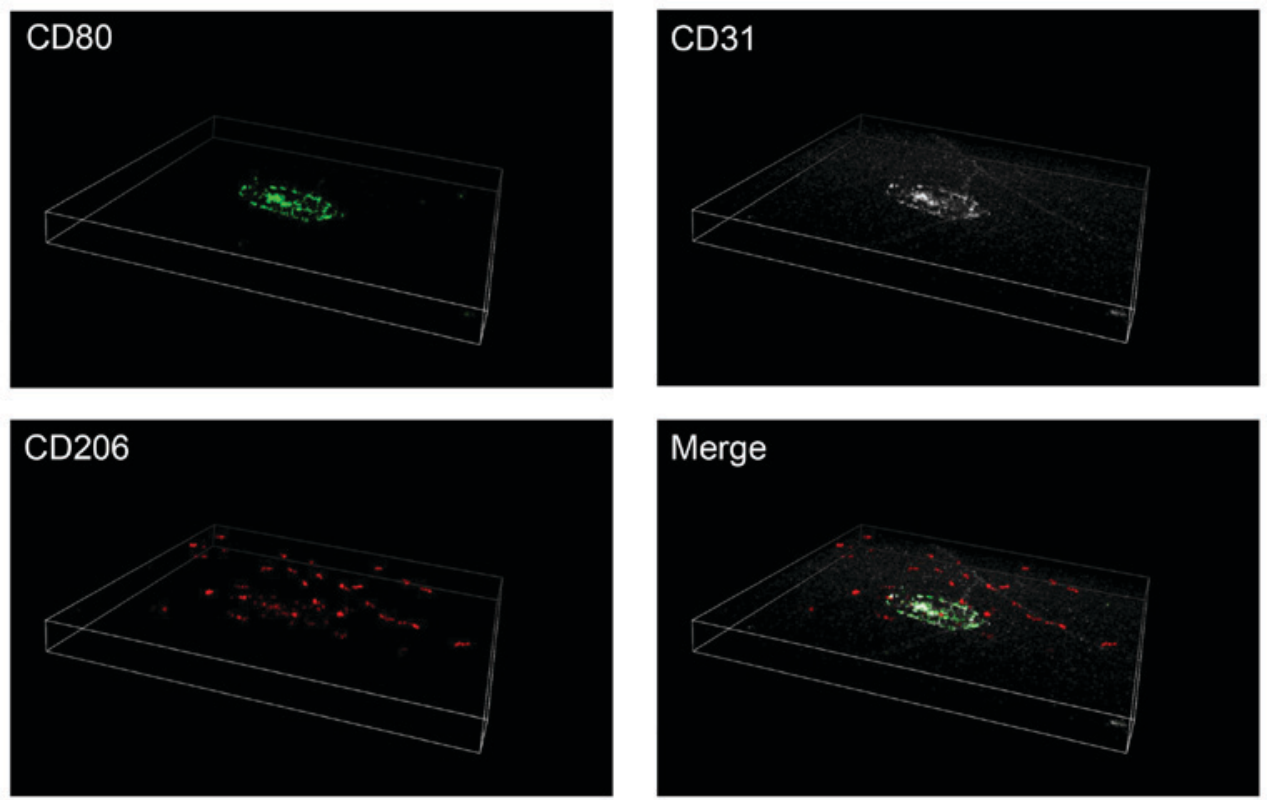

Figure 4. Three dimensional images of M1 and M2 macrophages. Immunofluorescence triple staining for CD80 (green), CD31 (white) and CD206 (red), in flat-mounted posterior segments of the eyes, with intact retina and choroid of CNV eyes at day 3. Numerous CD206-positive cells were observed in the retina, whereas CD80-positive cells were primarily located around the laser-induced CNVs. Magnification, x20. CD, cluster of differentiation; CNV, choroidal neovascularization.

A

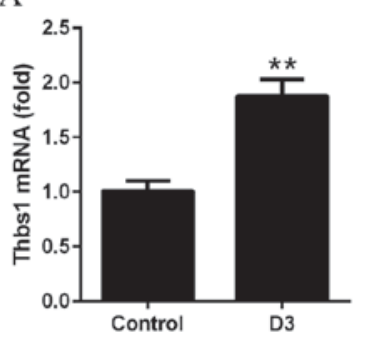

B

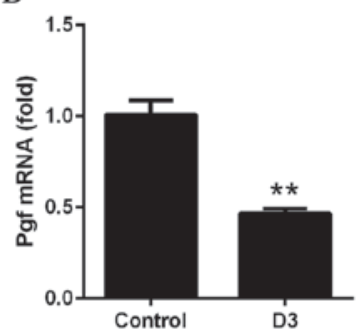

C

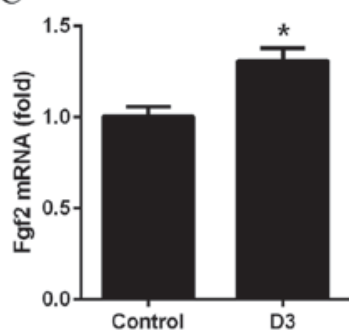

D

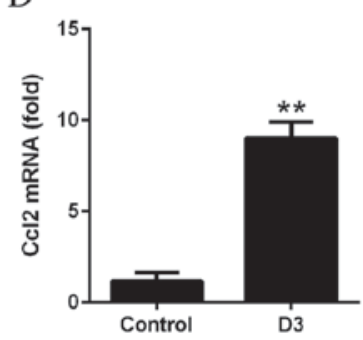

RPE-Choroid

E

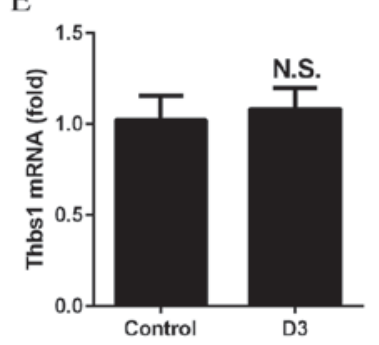

F

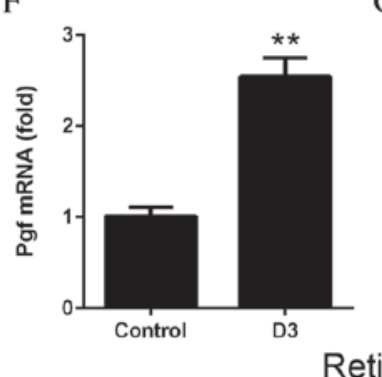

G

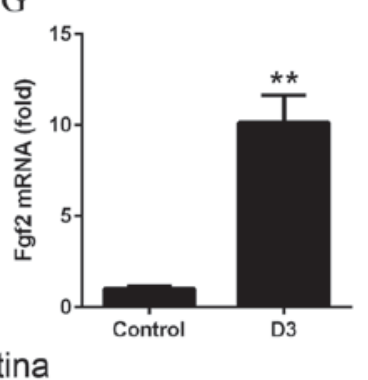

H

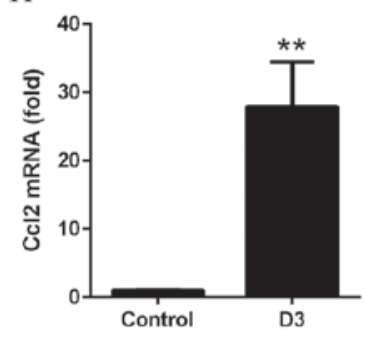

Figure 5. mRNA expression levels of angiogenic factors, as determined by reverse transcription-quantitative polymerase chain reaction. RPE-choroid complexes or retinas were harvested from mice 3 days following laser-induced choroidal neovascularization. In the RPE-choroid complexes, the mRNA expression levels of (A) Thbs1 were significantly increased following laser treatment, compared with untreated control tissues. Expression levels of (B) Pgf were decreased, (C) Fgf2 were increased and (D) Ccl2 were increased. In the retinas, no significant differences were observed in mRNA expression levels of (E) Thbs1 between control and laser-treated tissues; however, the mRNA expression levels of (F) Pgf, (G) Fgf2 and (H) Ccl2 were all significantly increased following laser treatment. Data are expressed as the mean \pm standard error $(n=4) .{ }^{* *} \mathrm{P}<0.01$ and ${ }^{*} \mathrm{P}<0.05$ vs. control. RPE, retinal pigment epithelium; Thbs1, thrombospondin 1; Pgf, placental growth factor; Fgf2, basic fibroblast growth; Ccl2, monocyte chemoattractant protein-1; D, day; NS, non-significant.

markers increased significantly in the RPE-choroid and retina following laser photocoagulation in a mouse model of CNV. However, the distributions of the M1 and M2 macrophages differed; M1 macrophages were detected to a greater extent in the RPE-choroid, whereas M2 macrophages were primarily located in the retina.

The expression of THBS1 has been demonstrated to be increased in M1 macrophages compared with monocytes, 
whereas M2 macrophages produce greater quantities of FGF2, PGF and CCL2 (14). It has been reported that THBS1 is an inhibitor of neovascularization in tumors (28). In the present study, Thbs1 mRNA expression levels increased significantly in the RPE-choroid complexes, consistent with the predominance of M1 macrophages around the laser-induced areas. By contrast, Fgf2 and Pgf mRNA expression levels increased primarily in the retinas, which is consistent with the presence of greater numbers of M2 macrophages in the retinas of the CNV model. Ccl 2 mRNA expression levels increased markedly in the retina and RPE-choroid complex, which may be due to the fact it is associated with the recruitment of the two macrophage subsets. These data further support the results of the immunofluorescence staining demonstrating the differential distribution of M1 and M2 macrophages.

Caicedo et al (7) reported that bone marrow-derived F4/80-positive macrophages infiltrated retinas and activated Müller cells leading to photoreceptor degeneration in a mouse model of CNV. Evidence suggests that retinal microglia and macrophages may migrate from the inner layer to the subretinal space to infiltrate the CNVs (29-31). These pathological alterations are essential for the development of CNVs, indicating that retinal microglia and macrophages may have a marked effect on the pathogenesis of CNVs. As M2 macrophages were the predominant subtype in the retinas in the present study, the M2 phenotype may comprise a large number of the macrophages and microglia observed in the retina in previous studies (7,29-31).

Retinal degeneration is associated with the development of CNVs in a rat model (32), indicating that retinal pathologies are relevant to the development of CNVs. M1 and M2 macrophages secrete different levels of molecules, including THBS1, FGF2, PGF and CCL2, as demonstrated in the present study, and it is possible that the function of each phenotype is partially based on these secreted molecules. M2 macrophages in the retina may therefore be relevant to the pathogenesis of CNVs. By contrast, M1 macrophages were located primarily in the RPE-choroid and may have a more direct effect in inhibiting the development of CNVs. There may be a conversion from M2 to M1 following the migration of macrophages from the retina to the choroid around the CNVs.

Macrophages are recognized to exist as two distinct subtypes, M1 pro-inflammatory and M2 anti-inflammatory $(8,9,14)$. In addition, it has been reported that monocytes/ macrophages may be differentiated by their expression of phenotypic markers, including $\mathrm{C}-\mathrm{C}$ chemokine receptor $2^{+}$Ly6 $\mathrm{C}^{\text {high }}$ (pro-inflammatory) and $\mathrm{CX} 3 \mathrm{C}$ chemokine receptor $1^{+}{ }^{2}$ 66 $C^{\text {low }}$ (anti-inflammatory) (33-35). Therefore, further studies are required to determine the contribution of these monocyte subsets to laser-induced CNVs.

In conclusion, the results of the present study demonstrated that M1 and M2 macrophages are recruited in response to laser-induced CNV. However, the distribution of these two cell subtypes differed; M1 macrophages were present primarily in RPE-choroid and M2 macrophages in the retina. Therefore, M1 macrophages may be more directly involved in laser-induced CNVs. These findings support and expand upon the results of previous studies on the functional roles of M1 and M2 macrophages in CNVs.

\section{Acknowledgements}

The present study was supported in part by the Japan Society for the Promotion of Science Grants-in-Aid for Scientific Research (B; grant nos. 15H04995 and 26293374), Grants-in-Aid for Challenging Exploratory Research (grant no. 16K15734), the Takeda Science Foundation and the China Scholarship Council (to Y.Z.). The authors thank Ms. Masayo Eto, Ms. Kinuko Sasada, and Ms. Hiroko Miura (Kyushu University) for their excellent technical assistance.

\section{References}

1. Klein R, Peto T, Bird A and Vannewkirk MR: The epidemiology of age-related macular degeneration. Am J Ophthalmol 137: 486-495, 2004.

2. Wong IY, Koo SC and Chan CW: Prevention of age-related macular degeneration. Int Ophthalmol 31: 73-82, 2011.

3. Ambati J, Ambati BK, Yoo SH, Ianchulev S and Adamis AP: Age-related macular degeneration: Etiology, pathogenesis, and therapeutic strategies. Surv Ophthalmol 48: 257-293, 2003.

4. Lambert V, Lecomte J, Hansen S, Blacher S, Gonzalez ML, Struman I, Sounni NE, Rozet E, de Tullio P, Foidart JM, et al: Laser-induced choroidal neovascularization model to study age-related macular degeneration in mice. Nat Protoc 8: 2197-2211, 2013.

5. Espinosa-Heidmann DG, Suner IJ, Hernandez EP, Monroy D, Csaky KG and Cousins SW: Macrophage depletion diminishes lesion size and severity in experimental choroidal neovascularization. Invest Ophthalmol Vis Sci 44: 3586-3592, 2003.

6. Sakurai E, Anand A, Ambati BK, van Rooijen N and Ambati J: Macrophage depletion inhibits experimental choroidal neovascularization. Invest Ophthalmol Vis Sci 44: 3578-3585, 2003.

7. Caicedo A, Espinosa-Heidmann DG, Pina Y, Hernandez EP and Cousins SW: Blood-derived macrophages infiltrate the retina and activate Muller glial cells under experimental choroidal neovascularization. Exp Eye Res 81: 38-47, 2005.

8. Mantovani A, Sica A, Sozzani S, Allavena P, Vecchi A and Locati M: The chemokine system in diverse forms of macrophage activation and polarization. Trends Immunol 25: 677-686, 2004.

9. Martinez FO, Sica A, Mantovani A and Locati M: Macrophage activation and polarization. Front Biosci 13: 453-461, 2008.

10. Mahdavian Delavary B, van der Veer WM, van Egmond M, Niessen FB and Beelen RHJ: Macrophages in skin injury and repair. Immunobiology 216: 753-762, 2011.

11. Gordon S and Martinez FO: Alternative activation of macrophages: Mechanism and functions. Immunity 32: 593-604, 2010.

12. Mantovani A, Schioppa T, Porta C, Allavena P and Sica A: Role of tumor-associated macrophages in tumor progression and invasion. Cancer Metastasis Rev 25: 315-322, 2006.

13. Ho VW and Sly LM: Derivation and characterization of murine alternatively activated (M2) macrophages. Methods Mol Biol 531: 173-185, 2009.

14. Jetten N, Verbruggen S, Gijbels MJ, Post MJ, De Winther MP and Donners MM: Anti-inflammatory M2, but not pro-inflammatory M1 macrophages promote angiogenesis in vivo. Angiogenesis 17: 109-118, 2014.

15. Cao X, Shen D, Patel MM, Tuo J, Johnson TM, Olsen TW and Chan CC: Macrophage polarization in the maculae of age-related macular degeneration: A pilot study. Pathol Int 61: 528-535, 2011.

16. Zhou Y, Yoshida S, Nakao S, Yoshimura T, Kobayashi Y, Nakama T, Kubo Y, Miyawaki K, Yamaguchi M,Ishikawa K, et al: M2 macrophages enhance pathological neovascularization in the mouse model of oxygen-induced retinopathy. Invest Ophthalmol Vis Sci 56: 4767-4777, 2015.

17. Zandi S, Nakao S, Chun KH, Fiorina P, Sun D, Arita R, Zhao M, Kim E, Schueller O, Campbell S, et al: ROCK-isoform-specific polarization of macrophages associated with age-related macular degeneration. Cell Rep 10: 1173-1186, 2015.

18. Zhang H, Yang Y, Takeda A, Yoshimura T, Oshima Y, Sonoda KH and Ishibashi T: A novel platelet-activating factor receptor antagonist inhibits choroidal neovascularization and subretinal fibrosis. PLoS One 8: e68173, 2013.

19. Nakama T, Yoshida S, Ishikawa K, Kobayashi Y, Zhou Y, Nakao S, Sassa Y, Oshima Y, Takao K, Shimahara A, et al: Inhibition of choroidal fibrovascular membrane formation by new class of RNA interference therapeutic agent targeting periostin. Gene Ther 22: 127-137, 2015. 
20. Ishikawa K, Yoshida S, Kadota K, Nakamura T, Niiro H, Arakawa S, Yoshida A, Akashi K and Ishibashi T: Gene expression profile of hyperoxic and hypoxic retinas in a mouse model of oxygen-induced retinopathy. Invest Ophthalmol Vis Sci 51: 4307-4319, 2010.

21. Li W, Katz BP and Spinola SM: Haemophilus ducreyi-induced interleukin-10 promotes a mixed M1 and M2 activation program in human macrophages. Infect Immun 80: 4426-4434, 2012.

22. Stein M, Keshav S, Harris N and Gordon S: Interleukin 4 potently enhances murine macrophage mannose receptor activity: A marker of alternative immunologic macrophage activation. J Exp Med 176: 287-292, 1992.

23. Bouhlel MA, Derudas B, Rigamonti E, Dièvart R, Brozek J, Haulon S, Zawadzki C, Jude B, Torpier G, Marx N, et al: PPARgamma activation primes human monocytes into alternative M2 macrophages with anti-inflammatory properties. Cell Metab 6: 137-143, 2007.

24. Livak KJ and Schmittgen TD: Analysis of relative gene expression data using real-time quantitative PCR and the 2(-Delta Delta C(T)) Method. Methods 25: 402-408, 2001.

25. Ishikawa K, Yoshida S, Nakao S, Sassa Y, Asato R, Kohno R, Arima M, Kita T, Yoshida A, Ohuchida K and Ishibashi T: Bone marrow-derived monocyte lineage cells recruited by MIP-1 $\beta$ promote physiological revascularization in mouse model of oxygen-induced retinopathy. Lab Invest 92: 91-101, 2012.

26. Arima M, Yoshida S, Nakama T, Ishikawa $K$, Nakao $S$, Yoshimura T, Asato R, Sassa Y, Kita T, Enaida H, et al: Involvement of periostin in regression of hyaloidvascular system during ocular development. Invest Ophthalmol Vis Sci 53: 6495-6503, 2012.

27. Ishikawa $K$, Yoshida $S$, Nakao S, Nakama T, Kita T, Asato R, Sassa Y, Arita R, Miyazaki M, Enaida H, et al: Periostin promotes the generation of fibrous membranes in proliferative vitreoretinopathy. FASEB J 28: 131-142, 2014.
28. Kazerounian S, Yee KO and Lawler J: Thrombospondins in cancer. Cell Mol Life Sci 65: 700-712, 2008.

29. Combadiére C, Feumi C, Raoul W, Keller N, Rodéro M, Pézard A, Lavalette S, Houssier M, Jonet L, Picard E, et al: CX3CR1-dependent subretinal microglia cell accumulation is associated with cardinal features of age-related macular degeneration. J Clin Invest 117: 2920-2928, 2007.

30. Ma W, Zhao L, Fontainhas AM, Fariss RN and Wong WT: Microglia in the mouse retina alter the structure and function of retinal pigmented epithelial cells: A potential cellular interaction relevant to AMD. PLoS One 4: e7945, 2009.

31. Huang H, Parlier R, Shen JK, Lutty GA and Vinores SA: VEGF receptor blockade markedly reduces retinal microglia/ macrophage infiltration into laser-induced CNV. PLoS One 8: e71808, 2013.

32. Albert DM, Neekhra A, Wang S, Darjatmoko SR, Sorenson CM, Dubielzig RR and Sheibani N: Development of choroidal neovascularization in rats with advanced intense cyclic light-induced retinal degeneration. Arch Ophthalmol 128: 212-222, 2010

33. Auffray C, Fogg DK, Narni-Mancinelli E, Senechal B, Trouillet C, Saederup N, Leemput J, Bigot K, Campisi L, Abitbol M, et al: CX3CR1 ${ }^{+} \mathrm{CD} 115^{+} \mathrm{CD} 135^{+}$common macrophage/DC precursors and the role of CX3CR1 in their response to inflammation. J Exp Med 206: 595-606, 2009.

34. Nahrendorf M, Swirski FK, Aikawa E, Stangenberg L, Wurdinger T, Figueiredo JL, Libby P, Weissleder R and Pittet MJ: The healing myocardium sequentially mobilizes two monocyte subsets with divergent and complementary functions. J Exp Med 204: 3037-3047, 2007.

35. Benakis C, Garcia-Bonilla L, Iadecola C and Anrather J: The role of microglia and myeloid immune cells in acute cerebral ischemia. Front Cell Neurosci 8: 461, 2015. 